\title{
镀液流动对泡沫炭真空超声化学镀铜的影响
}

\author{
杨 柳 ${ }^{1}$, 刘秀军 ${ }^{1}$, 李同起 ${ }^{2}$, 韩瑞连 ${ }^{1}$, 冯志海 ${ }^{2}$
}

(1. 天津工业大学 环境与化学工程学院, 天津 300387 ; 2. 航天材料及工艺研究所 先进功能复合材料技术国防科 技重点实验室, 北京 100076)

摘 要: 真空超声辅助化学镀铜, 可以在大体积泡沫炭内部孔隙表面镀铜, 但沉铜分布不均。在此基础上, 本研究强 制镀液在泡沫炭内部充分流动，并探究流动方式对镀层性能的影响。相比于镀液单向流动，镀液双向流动实现了铜 增量的均匀分布, 轴向切片铜的增重率超过 $7.10 \%$, 整块泡沫炭铜的增重率为 $7.68 \%$; 镀层均匀致密, 平整光滑，厚 度超过 $5 \mu \mathrm{m}$, 各向分布差异很小; 镀层无 $\mathrm{CuO} 、 \mathrm{Cu}_{2} \mathrm{O}$ 杂质; 机械性能、电学性能都得到均匀地提高, 压缩强度从 $0.70 \mathrm{MPa}$ 增大至 $1.54 \mathrm{MPa}$ ，电导率从 $700 \mathrm{~S} / \mathrm{cm}$ 增加至 $1724 \mathrm{~S} / \mathrm{cm}$ 。

关 键 词: 流动镀; 化学镀铜; 泡沫炭; 真空辅助

中图分类号: TB333 文献标识码: A

\section{Effect of Plating Solution Flow Pattern on Preparation of Electroless Copper- Plated Carbon Foam under Vacuum and Ultrasonic Conditions}

\author{
YANG Liu ${ }^{1}$, LIU Xiu-Jun ${ }^{1}$, LI Tong-Qi ${ }^{2}$, HAN Rui-Lian ${ }^{1}$, FENG Zhi-Hai ${ }^{2}$
}

(1. School of Environmental and Chemical Engineering, Tianjin Polytechnic University, Tianjin 300387, China; 2. National Key Laboratory of Advanced Functional Composite Materials, Aerospace Research Institute of Materials and Processing Technology, Beijing 100076, China)

\begin{abstract}
Electroless plating with assistance of vacuum and ultrasonic is an effective method to coat copper onto the inner surface of bulk carbon foam. However, it is difficult to obtain uniform copper coating. In present work, the copper coating's uniformity was improved by forcing plating solution inside the carbon foam on basis of vacuum and ultrasonic assistance during eletroless plating process. And effect of the flow pattern on plated copper's properties was investigated. Compared with one-direction flow of plating solution, a bidirectional flow made the weight gain rate of single cut piece $>7.10 \%$ and the total weight increased up to $7.68 \%$. The copper coating at thickness $>5 \mu \mathrm{m}$; with better uniformity was compact, smooth and no $\mathrm{CuO}$ or $\mathrm{Cu}_{2} \mathrm{O}$. The mechanical and electrical properties of $\mathrm{Cu} / \mathrm{carbon}$ foam were evenly enhanced. The compression strength was increased from $0.70 \mathrm{MPa}$ to $1.54 \mathrm{MPa}$, and the conductivity from $700 \mathrm{~S} / \mathrm{cm}$ to $1724 \mathrm{~S} / \mathrm{cm}$.
\end{abstract}

Key words: plating solution flow; electroless copper plating; carbon foam; vacuum assisted

泡沫炭是一种新型多孔炭材料, 具有低密度、 高开孔率 ${ }^{[1]}$ 、高强度、高导热导电性 ${ }^{[2-3]}$ 、抗冲击、 吸波和降噪等优异性能 ${ }^{[4]}$, 在航空航天、火箭、轮船
和催化剂载体等领域 ${ }^{[5-6]}$ 应用前景广阔。但泡沫炭的 导热性能、机械性能和电学性能较差, 限制了其进 一步应用。众多研究学者试图对泡沫炭进行改性研 
究 ${ }^{[7-8]}$ 。Lafdi 等 ${ }^{[9-11]}$ 选取网状玻璃态泡沫炭作基体, 采用电镀铜法制备了泡沫铜材料, 实验表明泡沫炭 内外表面镀层均匀致密, 并可通过调节施镀时间来 控制镀铜泡沫炭开孔率、导热率和机械强度, 镀层 厚度小于 $1 \mu \mathrm{m}$ 。

化学镀铜具有能耗低、包覆性好、附着力强、 抗腐蚀和耐磨损优异性能 ${ }^{[12-13]}$, 已成为泡沫炭改 性的新亮点。超声加速氢气脱离孔隙表面, 不仅可 以提高镀层致密性, 而且可以减小界面浓差极化, 提高镀液稳定性。赵鹏等 ${ }^{[14]}$ 在聚氨酯泡沫塑料化 学镀铜过程中辅以超声作用, 超声空化作用增加 了两相接触, 促进了溶液中 $\mathrm{Cu}^{2+}$ 向基体表面迁移, 增大了镀层厚度和镀铜深度。真空可以增大压强 差, 一方面将部分氢气抽离孔隙表面, 另一方面 控制镀液流动速率。杨春光等 ${ }^{[15]}$ 定量分析了气体 在非金属材料内部、表面、真空室内的扩散机理, 氢气在泡沫炭孔隙表面脱附抽离可以用其吸附扩 散模型进行解释。翟丽丽等 ${ }^{[16-17]}$ 引入真空超声共 辅助, 在中间相沥青基泡沫炭内外表面化学镀铜 方面取得一定突破, 提高了泡沫炭内部镀铜效果, 镀层厚度超过 $10 \mu \mathrm{m}$, 但沉铜分布、环保性等仍待 进一步突破。

大体积泡沫炭由于孔径小、孔深大, 传统化学 镀方法难以实现其内部孔隙表面的镀铜, 即使采 用真空超声辅助沉积, 仍然在镀层厚度和镀层分 布等方面存在技术难题。本工作拟采用超声、真空、 镀液流动的方法进行辅助, 镀液的流动强化了界 面传质, 成功实现了泡沫炭内部孔隙表面镀铜的 均匀化, 极大地提高了大体积泡沫炭的机械性能 和电学性能。

\section{1 实验方法}

\section{1 实验材料}

实验材料选用中间相沥青基泡沫炭, 尺寸 $\phi 85 \mathrm{~mm} \times$ $100 \mathrm{~mm}$, 孔隙率 30\% 60\%, 孔径 150 300 $\mu \mathrm{m}$ 。

\section{2 实验装置}

为了解决大体积泡沫炭镀层分布不均匀的问题, 通过分析泡沫炭内外镀液存在的浓度梯度及化学镀 的反应机理, 设计了超声真空流动化学镀铜装置, 如图 1 所示。

对泡沫炭进行外表面密封包覆并固定, 强制镀 液优先在基体内部充分流动。装置包括真空原、超 声装置和过滤装置, 其中所采用的真空室压力为 0.04 0.06 MPa, 超声功率为 $100 \mathrm{~W} / 42 \mathrm{kHz}$ 。适当的

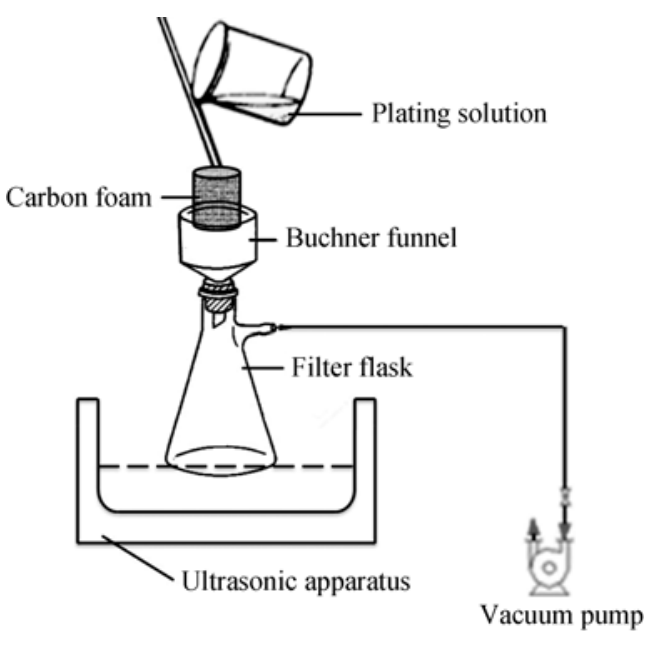

图 1 超声真空流动装置

Fig. 1 Schematic of vacuum and ultrasonic assisted flow electroless copper plating

流动可以降低界面的浓差极化 ${ }^{[14]}$, 提高镀铜效果 ${ }^{[18]}$ 。 过滤装置可以及时除去杂质粒子, 抑制镀液自分解。

\section{3 泡沫炭化学镀铜}

工艺流程: 除油 $\rightarrow$ 水洗 $\rightarrow$ 粗化 $\rightarrow$ 水洗 $\rightarrow$ 敏化 $\rightarrow$ 水洗 $\rightarrow$ 活化 $\rightarrow$ 水洗 $\rightarrow$ 流动化学镀铜, 以上步骤均在 流动方式下进行。

镀液组成及工艺条件如表 1 所示。

泡沫炭样品 CF1、CF2、CF3，分别采取不同的 流动方式进行真空超声辅助化学镀铜, 控制其他条 件不变, 观测不同流动方式对泡沫炭内部孔隙镀铜 效果的影响(规定 A 端为坐标原点)。

(1) CF1 直接置于镀液中进行反应;

(2) CF2 采取单向流动方式, 采用真空超声流动 装置进行反应，镀液始终自 $\mathrm{A}$ 向 $\mathrm{B}$ 流动;

(3) CF3 采取双向流动方式, 采用与 CF2 相同的

表 1 镀液组成及工艺条件

Table 1 Plating solution composition and process conditions

\begin{tabular}{lll}
\hline \multicolumn{1}{c}{ Steps } & \multicolumn{1}{c}{ Composition } & \multicolumn{1}{c}{ Conditions } \\
\hline Degreasing & $60 \mathrm{~g} / \mathrm{L} \mathrm{NaOH}^{\circ}$ & $50^{\circ} \mathrm{C}, 20 \mathrm{~min}$ \\
& $20 \mathrm{~g} / \mathrm{L} \mathrm{Na}_{2} \mathrm{CO}_{3}$ & \\
& $20 \mathrm{~g} / \mathrm{L} \mathrm{Na}_{3} \mathrm{PO}_{4}$ & \\
Roughening & $60 \% \mathrm{HNO}_{3}$ & $25^{\circ} \mathrm{C}, 20 \mathrm{~min}$ \\
Sensitization & $5 \% \mathrm{HCl}$ & $25 \mathrm{~min}$ \\
& $20 \mathrm{~g} / \mathrm{L} \mathrm{SnCl}{ }_{2}$ & \\
Activation & $30 \mathrm{~g} / \mathrm{L} \mathrm{AgNO}_{3}$ & $25 \mathrm{~min} ;$ \\
& & Vacuum, $10 \mathrm{~min}$ \\
Electroless & $20 \mathrm{~g} / \mathrm{L} \mathrm{CuSO}_{4} \cdot 5 \mathrm{H}_{2} \mathrm{O}$ & Room temperature \\
copper plating $26 \mathrm{~g} / \mathrm{L} \mathrm{Na}_{2} \mathrm{EDTA} \cdot 2 \mathrm{H}_{2} \mathrm{O}$ & $\mathrm{pH} 12.2-12.5$ \\
& $20 \mathrm{~g} / \mathrm{L} \mathrm{C} \mathrm{H}_{13} \mathrm{KO}_{8} \bullet 4 \mathrm{H}_{2} \mathrm{O}$ & Vacuum: \\
& $20 \mathrm{~mL} /{\mathrm{L} 2,2-\mathrm{Bipyridine}}$ & $0.04-0.06 \mathrm{MPa}$ \\
& $20 \mathrm{~mL} / \mathrm{L} \mathrm{methanal}(37 \%)$ & Ultrasonic: \\
& & $42 \mathrm{kHz}, 100 \mathrm{~W}$ \\
\hline
\end{tabular}


反应方法, 但镀液自 $\mathrm{A}$ 向 $\mathrm{B}$ 流动, 再自 $\mathrm{B}$ 向 $\mathrm{A}$ 流动 交替循环。

泡沫炭 CF1、CF2 和 CF3 反应一段时间后, 干 燥，根据图 2 所示自 $\mathrm{A}$ 端向 $\mathrm{B}$ 端平均分割为 5 份。 实验发现 CF1 内部几乎无铜覆盖, 因此本文将主要 研究单向流动方式和双向流动方式的影响。

\section{4 性能表征}

采用称重法测定镀铜反应的沉积速率, 将镀铜 泡沫炭放入足量硝酸中除铜, 称量样品除铜前后的 质量, 并由下式计算铜的沉积速率:

$$
v=\frac{m_{2}-m_{1}}{m_{1}} \times 100 \%
$$

式中: $v$ 为样品增重率; $m_{1} 、 m_{2}$ 分别为除铜前后样 品的质量, $\mathrm{g}$ 。

采用扫描电子显微镜(SEM)(JSM-7500F 型场发 射扫描电子显微镜)观测镀层微观表面形貌和厚度, 观测镀层厚度时, 样品需要采用树脂包埋。采用电 子万能材料试验机(CMT4503-5kN 电子万能材料试 验机) 测试镀铜泡沫炭的压缩强度, 加载速率为 $1 \mathrm{~mm} / \mathrm{min}$ 。采用四探针法(RTS-8 型四探针测试仪) 在室温下测量镀铜泡沫炭的电阻率。采用 $\mathrm{X}$ 射线衍 射仪(日本理学 Rigaku D/MAX-2500/PC 型 X 射线衍 射仪)分析镀层的晶体结构和微晶尺寸(采用 $\mathrm{Cu}$ 的 $\mathrm{K} \alpha$ 辐射, $\lambda=0.154056 \mathrm{~nm}$, 管压为 $4 \mathrm{kV}$, 管流为 $150 \mathrm{~mA}$ )。

\section{2 结果与讨论}

\section{1 镀液流动方式对铜增重率的影响}

图 3 是不同镀液流动方式铜增重率的轴向分布 曲线, 从图 3 可以看出, 单向流动镀下, 铜的增重率 自 $\mathrm{A}$ 端向 $\mathrm{B}$ 端存在明显的梯度, 到 $\mathrm{B}$ 端几乎可以忽 略; 双向流动镀下, 铜的增重率轴向差异很小, 并 且泡沫炭径向镀铜也较为均匀。因此, 采用双向流 动镀可以实现泡沫炭内部镀铜的均匀化, 而且提高

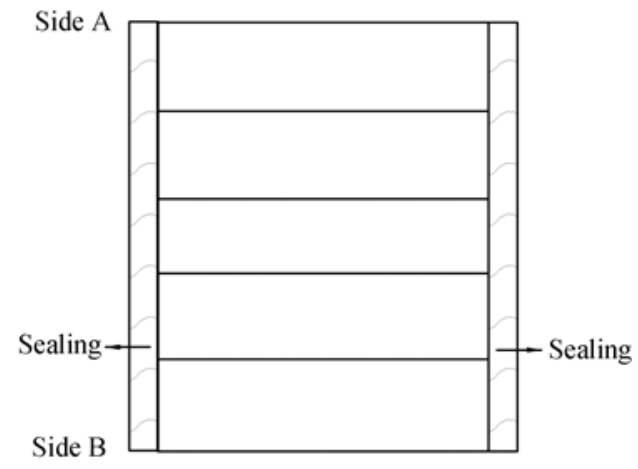

图 2 镀铜泡沫炭轴向切割示意图

Fig. 2 Ordinate cutting of $\mathrm{Cu} /$ carbon foam

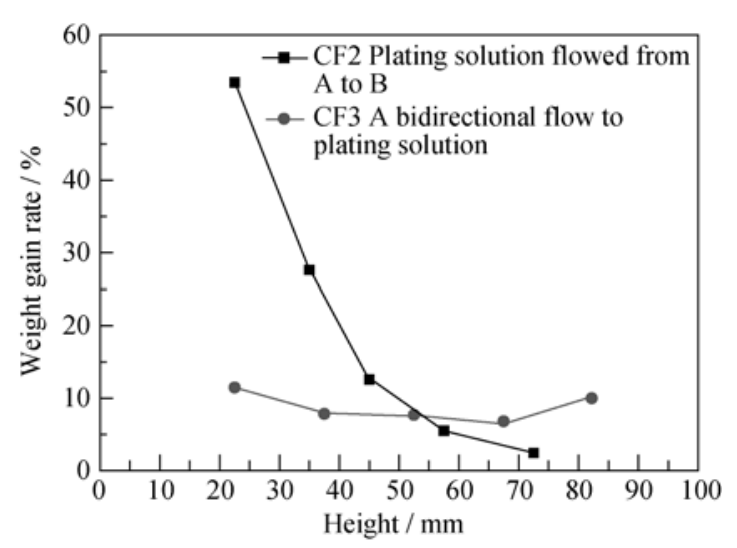

图 3 不同镀液流动方式铜增重率的轴向分布曲线

Fig. 3 Copper coating weight gain rate $v s$ height in different plating solution flow patterns

了大体积泡沫炭整体的铜增量, 轴向切片的铜增重 率超过 $7.10 \%$, 整块泡沫炭的铜增重率为 $7.68 \%$ 。

与直接置于镀液中反应相比较, 镀液流动时产 生向下的推动作用和真空作用, 使反应产生的氢气 被迅速地抽离孔隙表面并带出泡沫炭, 反应为

$$
\mathrm{Cu}^{2+}+2 \mathrm{HCHO}+4 \mathrm{OH}^{-} \rightarrow \mathrm{Cu}+2 \mathrm{HCOO}^{-}+\mathrm{H}_{2} \uparrow+2 \mathrm{H}_{2} \mathrm{O}
$$

镀液可直接与孔隙表面接触反应, 为铜在孔隙表面 进行沉积提供了可能。单向流动镀的轴向差异是由 于镀液每次进入泡沫炭优先经过 $\mathrm{A}$ 端, 镀液中各组 分浓度较高, 有利于铜的沉积; 随着镀液向 $\mathrm{B}$ 段流 动, 镀液中各组分浓度急剧下降, 主反应速率明显 降低, 副反应加剧, 逐渐难以沉铜; 也有可能是随 着反应的进行, $\mathrm{A}$ 端孔隙表面铜沉积较多阻塞了孔 隙, 阻碍镀液向 $\mathrm{B}$ 端流动, 如图 4 所示。双向流动 镀下, 由于镀液交替从 $\mathrm{A}$ 端和 $\mathrm{B}$ 端流动, $\mathrm{A} 、 \mathrm{~B}$ 两端 得到相同的机会与最佳组成的镀液接触, 有利于轴 向均匀地进行铜沉积; $\mathrm{A}$ 端铜的增量率略高于 $\mathrm{B}$ 端, 可能是因为泡沫炭制作过程中 $\mathrm{A}$ 端的孔隙较大, 镀 液的铜沉积的客观条件略好。

\section{2 镀液流动方式对镀层宏观表面形态的影响}

观察比较 CF2、CF3 轴向切片横截面, 宏观结 果见表 2 所示。分析表 2, 单向流动镀下, 镀层质量 自 $\mathrm{A}$ 端向 $\mathrm{B}$ 端有明显的梯度差异; 双向流动镀下, (a)

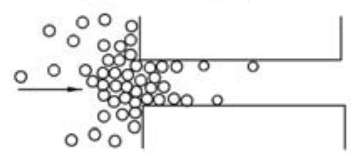

$\circ$

CF2: Plating solution flowed from A to B (a)

CF3: A bidirectional flow of plating solution(b) Remark: $\rightarrow$ Flow pattern of plating solution (b)

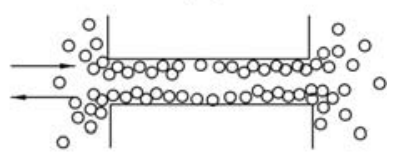

图 4 泡沫炭内部孔隙表面的铜沉积过程示意图

Fig. 4 Schematic of copper deposition on pore surface 
表 2 镀液流动方式对镀层宏观表面形态轴向分布的影响 (自 A 端向 B 端)

Table 2 Macroscopic morphology of copper coating vs. height in different plating solution flow patterns (From A to B)

\begin{tabular}{ccc}
\hline & CF2 & CF3 \\
\hline \multirow{2}{*}{ Color } & Bright red $\rightarrow$ Black & Copper red; \\
& Well-distributed \\
Compactness & Density $\downarrow$ & Uniform and dense; \\
& Porosity $\uparrow$ & Well-distributed \\
Smoothness & Leveling $\downarrow$ & Smoothy and flat; \\
& Surface roughness $\uparrow$ & Well-distributed \\
\hline
\end{tabular}

镀层颜色为亮铜色、致密均匀，平整光滑，梯度差异 很小，说明采用双向流动镀可以大大降低泡沫炭内 部镀层质量的差异。

与直接置于镀液中反应相比较, 流动镀中氢气 的及时抽走有效避免了 “氢脆现象”; 过滤装置将 副反应产生的杂质粒子如铜颗粒、氧化铜颗粒、氧 化亚铜颗粒 ${ }^{[19-20]}$ 及时除去, 提高镀层纯度, 从而大 大提高了泡沫炭内部镀层的厚度、颜色、致密性和 平整性。单向流动镀下, 自 $\mathrm{A}$ 端向 $\mathrm{B}$ 端镀层铜色越 来越浅、孔隙率增加、平整性下降，主要是因为沉 铜量下降; 并且镀液由 $\mathrm{A}$ 端向 $\mathrm{B}$ 端流动过程中，铜 盐浓度急剧降低，可能会导致副反应加剧，少量杂 质粒子与铜共沉积。双向流动镀下，镀液稳定，有效 抑制副反应的发生，故镀层质量好，镀液中铜的利 用率可达 $75 \%$ 以上。

\section{3 镀液流动方式对镀层微观表面形貌的影响}

图 5 为不同镀液流动方式下, 镀层微观表面形 貌轴向分布。单向流动镀下，距 $\mathrm{A}$ 端 $30 \mathrm{~mm}$ 以内，孔 隙表面被铜均匀包覆，表面形貌致密平整光滑; 距 离 $\mathrm{A}$ 端超过 $50 \mathrm{~mm}$ 的孔隙壁包覆不完全; 距离 $\mathrm{A}$ 端 $80 \mathrm{~mm}$ 左右基本无铜覆盖。双向流动镀下，孔隙表 面被铜均匀包覆，表面形貌致密平整光滑。这主要 是因为双向流动有效降低孔隙表面镀液的浓差极

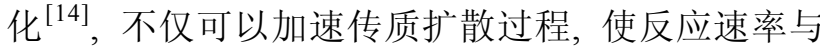
流动速率维持在一个相对稳定的范围，为铜沉积创 造条件; 还可以提高晶粒成核率，使镀层结晶的晶 粒细化和致密化 ${ }^{[18]}$ 。超声的空化作用将镀液中团聚 颗粒打碎成小颗粒，有利于铜粒子均匀沉积于不同 的催化中心 ${ }^{[21]}$, 提高镀层的致密性。

\section{4 镀液流动方式对镀层厚度的影响}

图 6 是不同镀液流动方式镀层厚度轴向分布, 由图 6 可以看出, 单向流动镀下, 在距 $\mathrm{A}$ 端 $30 \mathrm{~mm}$ 以内, 镀层厚度超过 $11 \mu \mathrm{m}$, 但沿轴向镀层厚度减 小很快; 距 $\mathrm{A}$ 端距离超过 $50 \mathrm{~mm}$, 镀层非常薄且不连

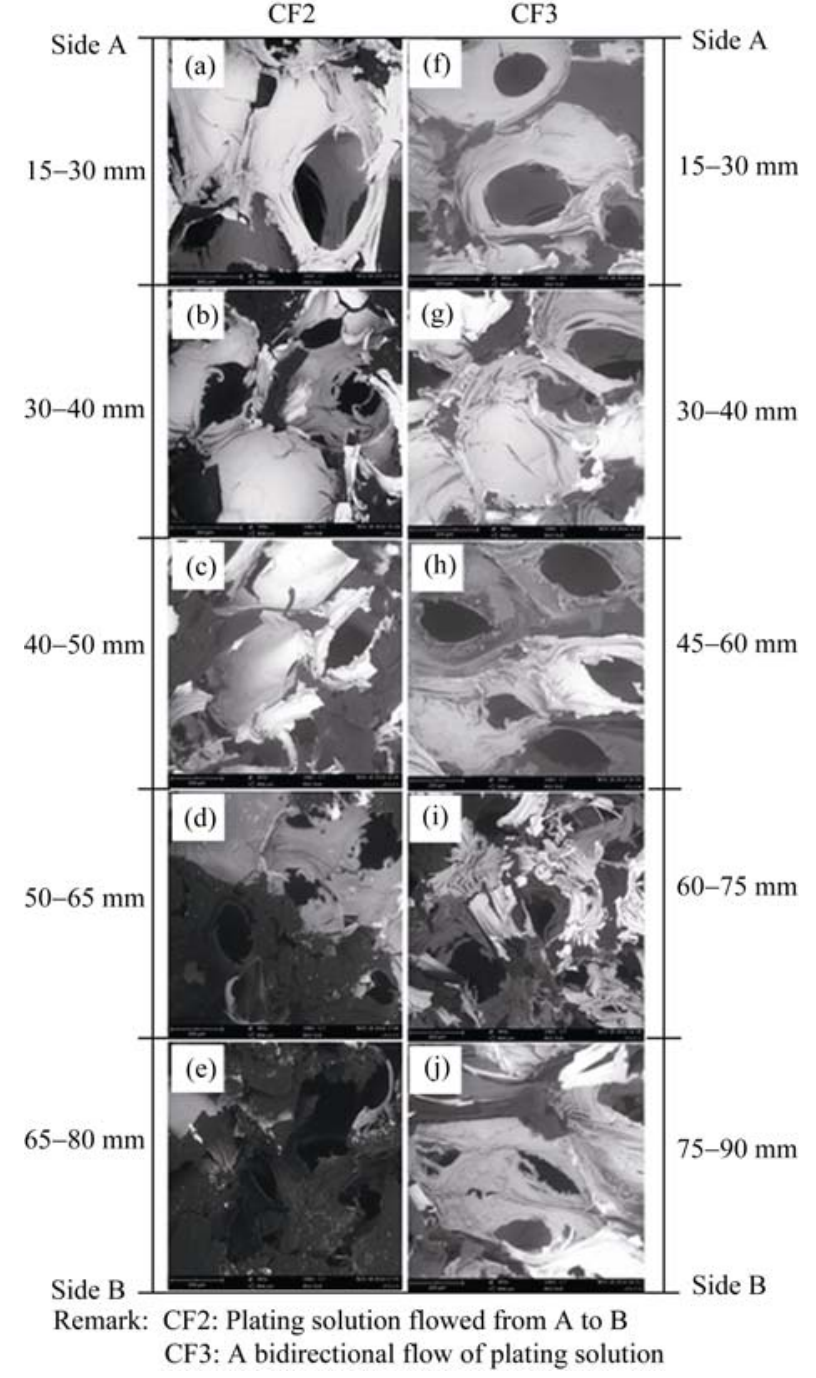

图 5 不同镀液流动方式镀层微观表面形貌轴向分布

Fig. 5 SEM images of copper coating $v$ s. height in different plating solution flow patterns

续。双向流动镀下，镀层厚度均匀，在 5 7 $\mu \mathrm{m}$ 之间。

\section{5 镀液流动方式对镀铜泡沫炭压缩强度的影响}

图 7 是不同镀液流动方式镀铜泡沫炭压缩强度 轴向分布，由图 7 可知，单向流动镀下，泡沫炭压缩 强度自 $\mathrm{A}$ 端向 $\mathrm{B}$ 端大幅度下降; 距 $\mathrm{A}$ 端距离超过 $50 \mathrm{~mm}$ ，几乎与未镀铜前无异。双向流动镀下，泡沫 炭压缩强度得到均匀地大幅度提高, 从 $0.70 \mathrm{MPa}$ 增 大至 $1.54 \mathrm{MPa}$ 。

在泡沫炭受压过程中，基体炭骨架成为支撑材 料的主要因素, 而骨架结构存在的裂缝和错位是材 料破坏的主要原因。双向流动镀下，连续致密的铜 镀层在泡沫炭㓞带及断裂处沉积、连接, 有效地阻 止裂纹在薄弱的孔壁或预存在的缺陷处发生集结, 弥补泡沫炭基体㓞带断裂对骨架强度的影响，增大 了泡沫炭基体在压缩时的阻力，从而使泡沫炭的压 缩强度得到大幅度提高。 


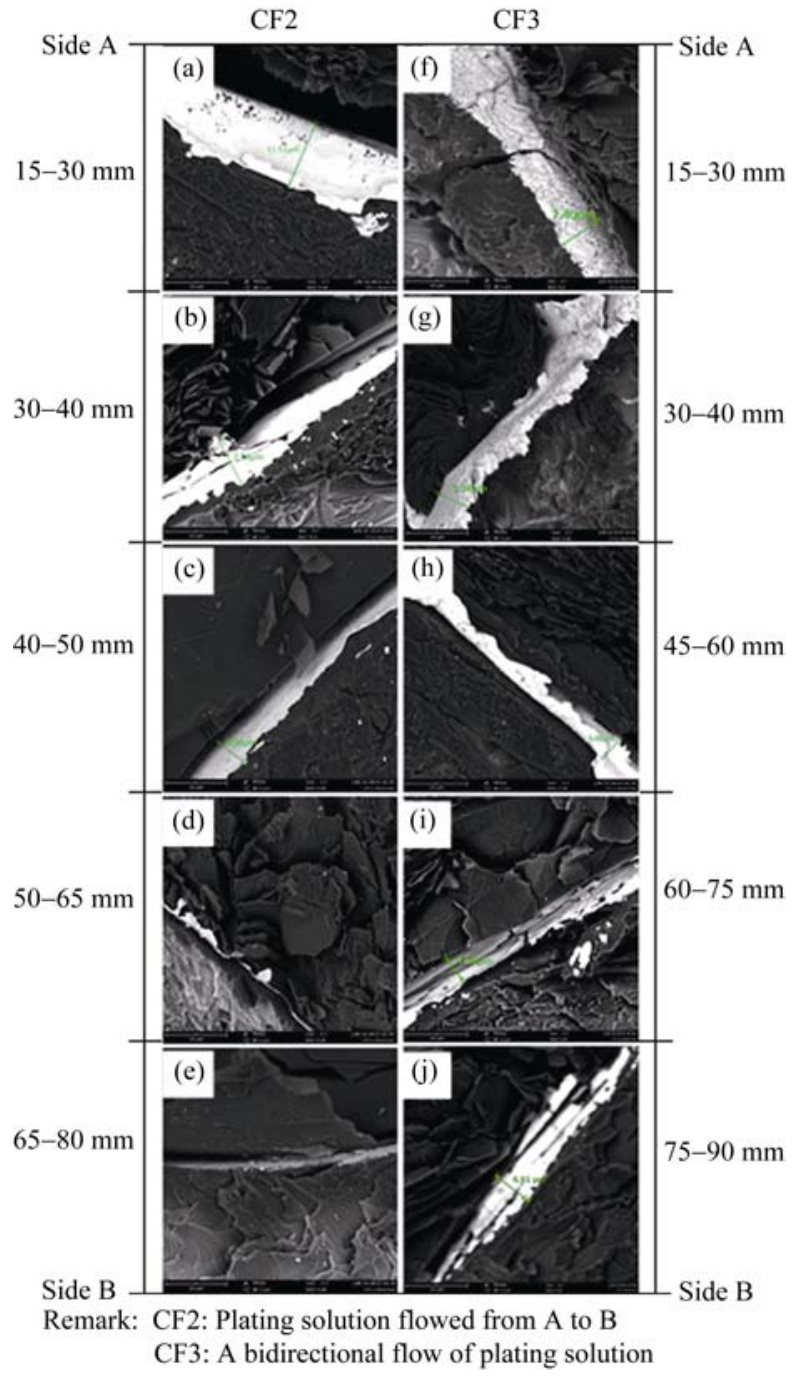

图 6 不同镀液流动方式镀层厚度轴向分布

Fig. 6 Coating thickness vs. height in different plating solution flow patterns

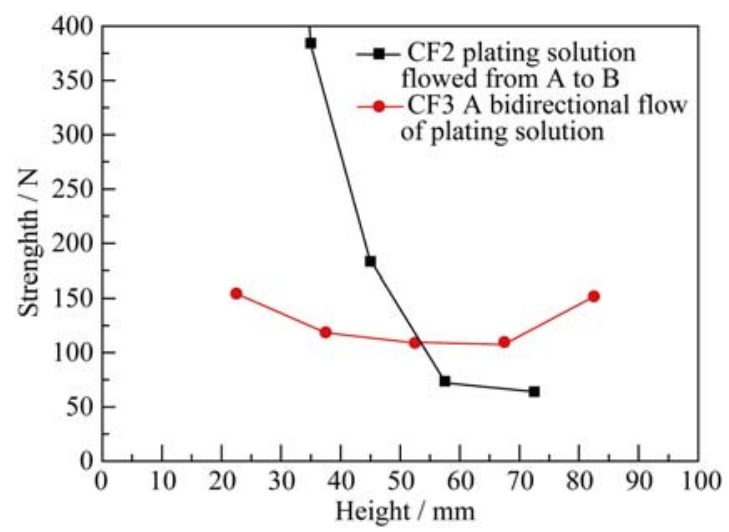

图 7 不同镀液流动方式镀铜泡沫炭压缩强度轴向分布

Fig. 7 Compressive strength of $\mathrm{Cu} /$ carbon foam $v s$ height in different plating solution flow pattern

\section{6 镀液流动方式对镀层电导率的影响}

图 8 是不同流动方式镀铜泡沫炭电导率轴向分

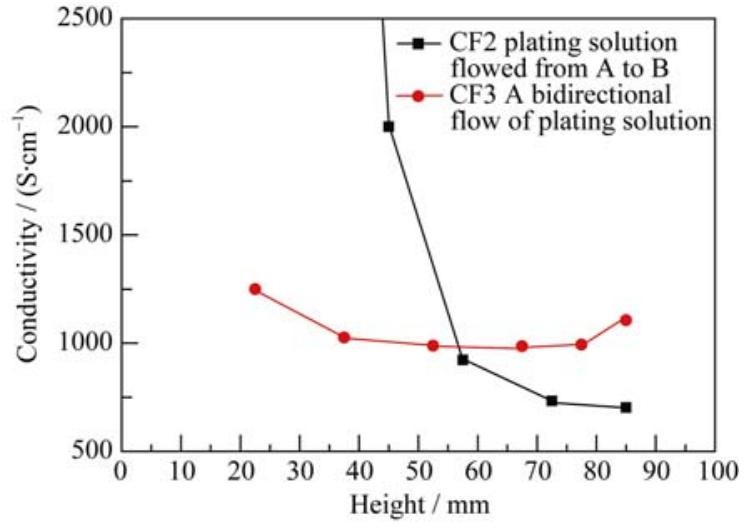

图 8 不同流动方式镀铜泡沫炭电导率轴向分布曲线

Fig. 8 Conductivity of copper coating $v s$ height in different plating flow patterns

布曲线, 从图 8 可以看出, 电导率对镀铜量比较敏感, 单向流动镀下, 距 A 端 $50 \mathrm{~mm}$ 以内, 电导率几乎呈直 线下降; $70 \mathrm{~mm}$ 以外几乎与未镀铜前无差异。双向流动 镀下，电导率都大于 $1000 \mathrm{~S} / \mathrm{cm}$, 轴向差异小。

均匀致密的镀层与泡沫炭基体实现机械锁合, 将泡沫炭韧带层及裂隙连为一体，这种结构为自由 电子在层间的传递提供了更好的路径，降低了镀层 电阻率。并且连接材料铜的电阻率远远小于泡沫炭, 因此实现了镀铜泡沫炭高电导率。

\section{7 双向流动镀中镀铜泡沫炭的晶体结构和 微晶尺寸}

图 9 是双向流动镀的镀铜泡沫炭的 XRD 图谱, 由图 9 看出, 泡沫炭内外表面均有铜沉积, 外表面 镀层分别在 $43.30^{\circ} 、 50.44^{\circ} 、 74.13^{\circ} 、 89.90^{\circ}$ 和 $95.10^{\circ}$ 出现了铜的(111)、(200)、(220)、(311)和(222)的衍射

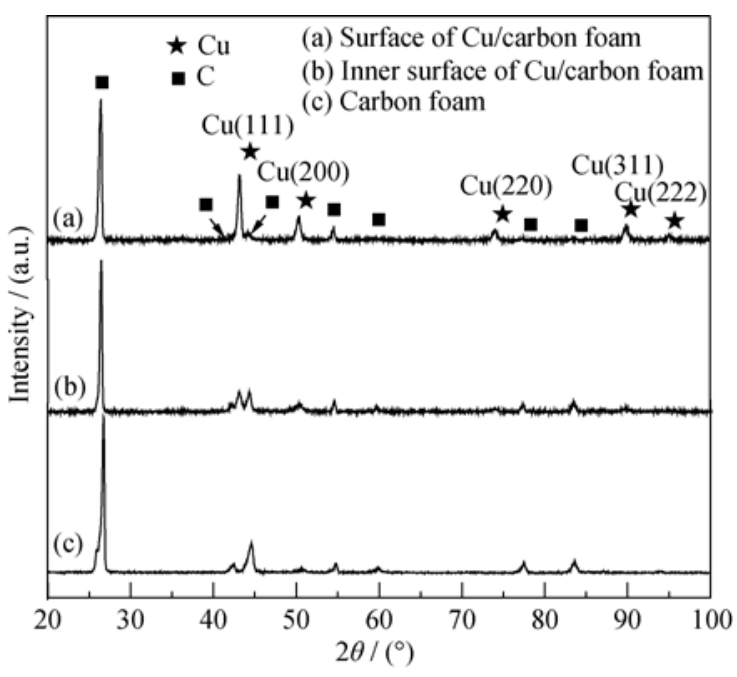

图 9 双向流动镀的镀铜泡沫炭的 XRD 图谱

Fig. 9 XRD patterns of $\mathrm{Cu} /$ carbon foam obtained at a bidirectional flow of plating solution 
峰; 内部孔隙表面的镀层的 (222)衍射峰完全消失。 镀层中无铜的氧化物和其它杂质出现。整个镀层均 为结晶铜, 且铜晶粒大小均匀, 晶粒尺寸为 $40.8 \mathrm{~nm}$ 。

\section{3 结论}

1) 真空超声辅助化学镀铜中, 不同流动方式镀 液决定泡沫炭内部孔壁镀铜效果。直接置于镀液中 进行反应, 基本无铜沉积; 单向流动镀, 沉铜量存在 明显的轴向梯度差异; 双向流动镀可以实现铜增量 的均匀分布, 且增重率在 $7.10 \%$ 以上。

2) 双向流动镀中, 泡沫炭内部孔壁镀层均匀致 密、光滑平整，厚度超过 $5 \mu \mathrm{m}$, 且各向分布变化很小; 镀层纯度高; 电学性能和机械性能得到均匀地改善, 电导率从 $700 \mathrm{~S} / \mathrm{cm}$ 增加至 $1774 \mathrm{~S} / \mathrm{cm}$, 压缩强度从 $0.70 \mathrm{MPa}$ 增大至 $1.54 \mathrm{MPa}$ 。

\section{参考文献:}

[1] LI T Q, WANG C Y. Preparation and structure characterization of mesophase pitch-based carbon foam. Journal of Inorganic Materials, 2005, 20(6): 1438-1444.

[2] KLETT J W, MCMILLAN A D, GALLEGO N C, et al. Effects of heat treatment conditions on the thermal properties of mesophase pitch-derived graphitic foams. Carbon, 2004, 42(8/9): 1849-1852.

[3] KLETT J, HARDY R, ROMINE E, et al. High-thermal-conductivity mesophase pitch-derived carbon foams: effect of precursor on structure and properties. Carbon, 2000, 38(7): 953-973.

[4] 王妹先. 中间相沥青基泡沫炭的制备及性能研究. 天津: 天津 大学硕士学位论文, 2008.

[5] SIHN S, ROY A K. Modeling and prediction of bulk properties of open-cell carbon foams. J. Mech. Phys. Solids. , 2004, 52(1): 167-191.

[6] WANG M X, WANG C Y, LI T Q, et al. Preparation of mesophase-pitch-based carbon foams at low pressures. Carbon, 2008, 46(1): 84-91.

[7] LI S Z, SONG Y Z, SONG Y, et al. Carbon foams with high compressive strength derived from mixtures of mesocarbon microbeads and mesophase pitch. Carbon, 2007, 45(10): 2092-2097.

[8] Vignoles G L, GaborieAu C, Delettrez S, et al.
Reinforced carbon foams prepared by chemical vapor infiltration: a process modeling approach. Surf. Coat. Tech. , 2008, 203(5/6/7): 510-515.

[9] LAFDI K, ALMAJALI M, HUZAYYIN O. Thermal properties of copper-coated carbon foams. Carbon, 2009, 47(11): 2620-2626.

[10] ALMAJALI M, LAFDI K, PRODHOMME P H, et al. Mechanical properties of copper-coated carbon foams. Carbon, 2010, 48(5): 1604-1608.

[11] ALMAJALI M, LAFDI K. Assessment of carbon foam geometry during copper coating. Carbon, 2010, 48(15): 4238-4247.

[12] BHAV SINGH B, BALASUBRAMANIAN M. Processing and properties of copper-coated carbon fibre reinforced aluminum alloy composites. J. Mater. Process. Technol. , 2009, 209(4): 2104-2110.

[13] CHE D, YAO G C, GAO Z K. A precious metal-free electroless technique for the deposition of copper on carbon fibers. Metall. Mater. Trans. , 2012, 43(11): 4194-4199.

[14] ZHAO P, PU Y P, HUANG C H, et al. Effect of adding ultrasonic on copper electroless plating of polyurethane foam. Materials Engineering, 2008(4): 43-46.

[15] YANG C G, XIAO Y M, CHEN N, et al. A comprehensive survey of development of outgassing model for nonmetal materials in vacuum. Vacuum, 2006, 43(3): 48-50.

[16] ZHAI L L, LIU X J, LI T Q, et al. Vacuum and ultrasonic co-assisted electroless copper plating on carbon foams. Vacuum, 2015, 114: 21-25.

[17] ZHAI L L, LIU X J, LI T Q, et al. Without activation formaldehyde electroless copper plating in graphite plate deep hole and silt. Materials Reviews, 2014, 28(8): 99-103.

[18] YU G, ZOU C, HU B N, et al. Research on preparation of $\mathrm{Cu}$-coated graphite powders with ultrasonic flow electroplating copper. Journal of Hunan University, 2011, 38(2): 60-64.

[19] 李宁. 化学镀实用技术. 北京: 化学工业出版社, 2004: 265-307.

[20] GUO Z C, LIU H K, WAN Z Y, et al. Present status and prospect of electroless plating deposits. Surface Technology, 1995, 24(6): $29-33$.

[21] CAO D, LI Z H. Preparation of siler-coated glass fiber by electroless plating with ultrasonic threatment. Materials Science and Engineering of Powder Metallurgy, 2009, 14(6): 422-426. 